\title{
Investigation of the heavy nuclei fission with anomalously high values of the fission fragments total kinetic energy
}

\author{
Vitaly Khryachkov^, Andrei Goverdovskii, Vladimir Ketlerov, Vecheslav Mitrofanov, \\ and Alexei Sergachev \\ Institute of Physics and Power Engineering, 1, Bondarenko sq., Obninsk 249030, Russia
}

\begin{abstract}
Binary fission of ${ }^{232} \mathrm{Th}$ and ${ }^{238} \mathrm{U}$ induced by fast neutrons were under intent investigation in the IPPE during recent years. These measurements were performed with a twin ionization chamber with Frisch grids. Signals from the detector were digitized for further processing with a specially developed software. It results in information of kinetic energies, masses, directions and Bragg curves of registered fission fragments. Total statistics of a few million fission events were collected during each experiment. It was discovered that for several combinations of fission fragment masses their total kinetic energy was very close to total free energy of the fissioning system. The probability of such fission events for the fast neutron induced fission was found to be much higher than for spontaneous fission of ${ }^{252} \mathrm{Cf}$ and thermal neutron induced fission of ${ }^{235} \mathrm{U}$. For experiments with ${ }^{238} \mathrm{U}$ target the energy of incident neutrons were $5 \mathrm{MeV}$ and $6.5 \mathrm{MeV}$. Close analysis of dependence of fission fragment distribution on compound nucleus excitation energy gave us some explanation of the phenomenon. It could be a process in highly excited compound nucleus which leads the fissioning system from the scission point into the fusion valley with high probability.
\end{abstract}

\section{Introduction}

We would like to point out some particular cases of nuclear fission when fission fragments appeared to have no excitation energy, at equilibrium deformation. In such cold-fragmentation events the total energy $Q^{*}$ transforms into the fragment kinetic energy $E k$ due to their Coulomb repulsion. Expression for total energy of the reaction is

$$
Q^{*}=Q+B n+E n,
$$

where $Q$ is the energy of nuclear fission, $B n$ is the neutron binding energy in the fissile nucleus, and $E n$ is the neutron kinetic energy.

This phenomenon was studied in several works (see, for example, [1-5]) and it is of great interest because we have a possibility to get information about the evolution of fissile system. Fission fragment kinetic energy can reach the maximum of possible values only if the fragments were produced almost at the saddle point. The probability of such fission events depends directly on the properties of the potential surface corresponding to the fissile system. In particular, the penetrability of the barrier between the fission and fusion valleys is of great importance. The dynamical component of the kinetic

${ }^{\star}$ e-mail: hva@ippe.ru 
energy depends on the deformation of the fissioning nucleus as it descends from the saddle point to the scission point. In the case of cold fragmentation this stage of the fission process is omitted, because of at the saddle point the nucleus transforms into two fission fragments in the ground state. They have no excitation energy and therefore cannot emit neutrons or gamma rays.

It is very interesting to compare the same fissile system at various values of excitation energies. By estimating the penetrability of the barrier between the fission and fusion valleys at the different excitation energies of the fissile nucleus we can get an idea of the parameters of this barrier.

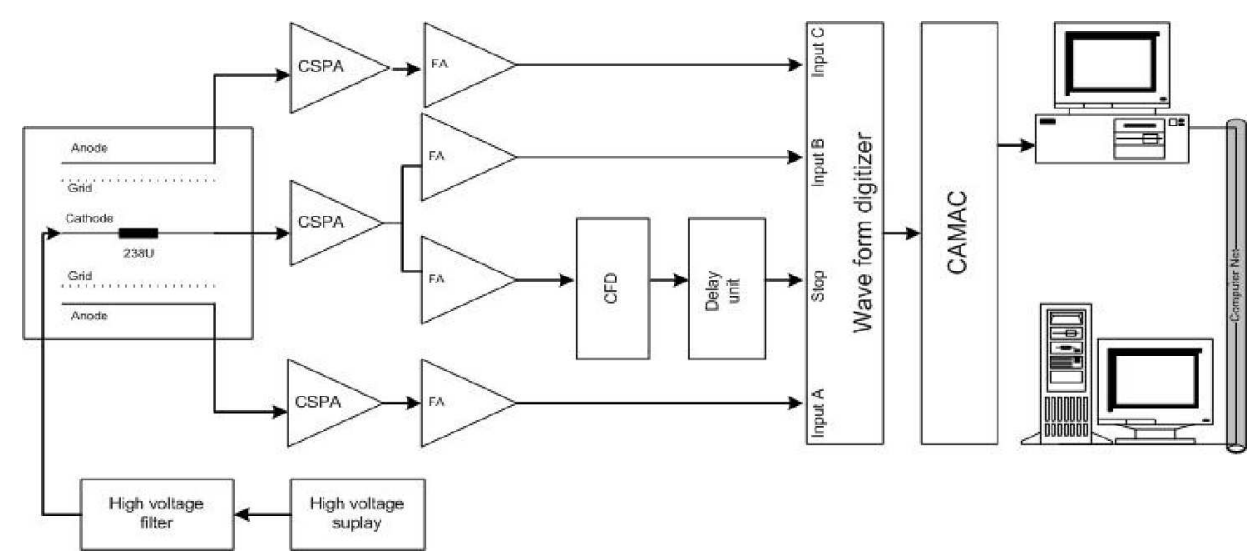

Figure 1. Layout of spectrometer electronics.
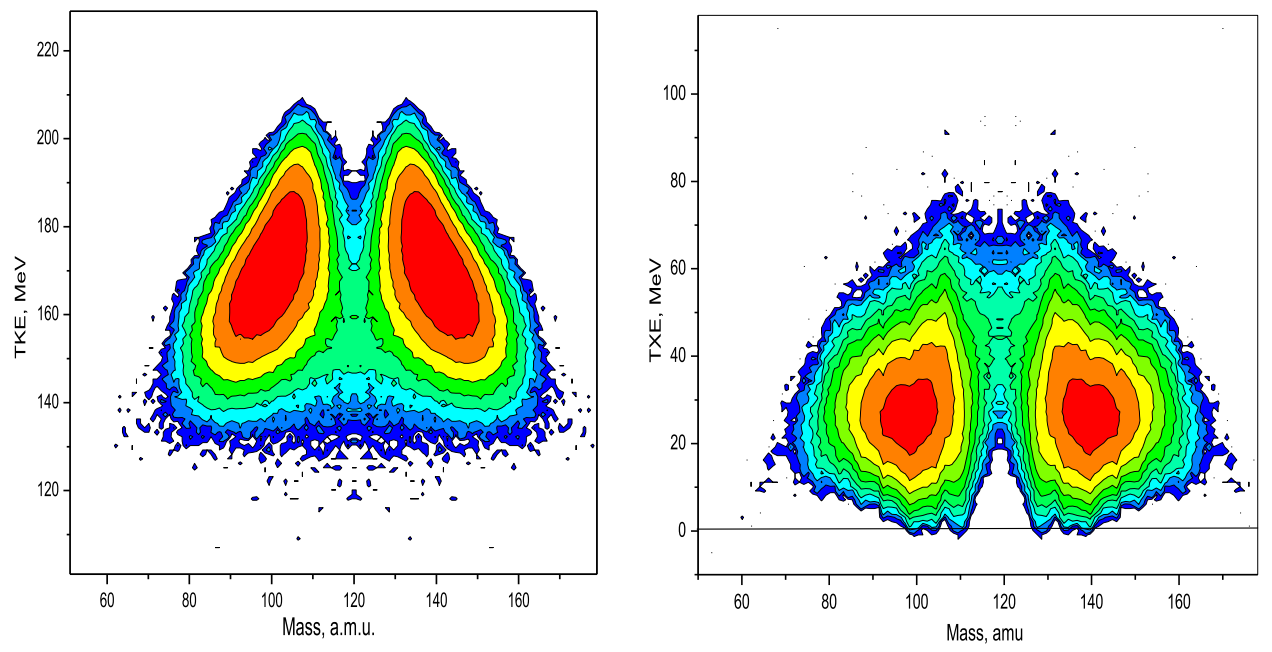

Figure 2. Distribution of ${ }^{239} \mathrm{U}$ fragment mass versus TKE (left) and TXE (right) for the incident neutron energy of $5 \mathrm{MeV}$. 

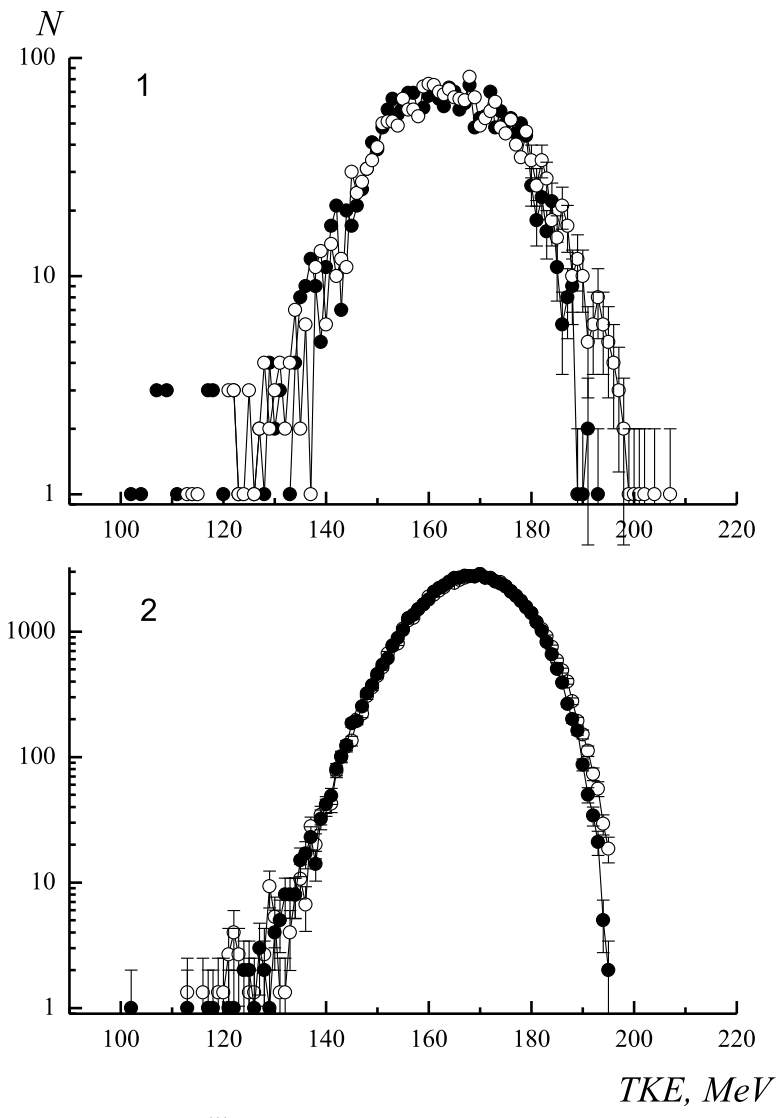

Figure 3. TKE distribution for ${ }^{239} \mathrm{U}$ fragment mass $123 \mathrm{amu}$ (1) $140 \mathrm{amu}$ (2). Full points - neutron energy $5 \mathrm{MeV}$, open points $-6.5 \mathrm{MeV}$.

\section{Experiment}

Fission fragments were detected in a twin ionization chamber with Frisch grids. The ${ }^{238} \mathrm{U}$ target of a diameter of $3 \mathrm{~cm}$ and a thickness of $100 \mu \mathrm{g} / \mathrm{cm}^{2}$ was mounted on the common cathode. It was prepared by vacuum sputtering of uranium fluoride onto $\mathrm{Al}_{2} \mathrm{O}_{3}$ backing having a thickness of $50 \mu \mathrm{g} / \mathrm{cm}^{2}$. In order to have good electric conductivity over the entire cathode surface the target was additionally coated with $50 \mu \mathrm{g} / \mathrm{cm}^{2}$ of gold. The schematics of the experimental facility is shown in Fig. 1. The signals from the anodes were collected as digital oscillograms of 512 points with a step time of $12.5 \mathrm{~ns}$ from the LeCroy 2262 pulse-shape digitizer on the data acquisition computer. Offline digital signal processing and analysis of saved oscillograms allows us to determine the kinetic energy, the mass, and the emission angle [4] of the pair of fission fragments. The energies of fission fragments were obtained with the corrections for the Frisch grid inefficiency [6], for the energy loss in the target [7], and for the pulse height defect [4]. Additional filter was performed for the pile-ups from alpha particles or scattered protons. 
The experiments were carried out in a neutron beam of the EG-1 accelerator of the Institute for Physics and Power Engineering (Obninsk, Russia). The reaction D $(\mathrm{d}, \mathrm{n})^{3} \mathrm{He}$ was used to obtain 5 and 6.5 MeV neutrons. The mean current at the target was $50 \mu \mathrm{A}$.

During a one month long measurement, we were able to accumulate and analyze 2.5 million digital oscillograms corresponding to ${ }^{239} \mathrm{U}$ fission events. In order to calibrate the primary distributions, we took data reported in [8], where one can find high precision mass and energy distributions for ${ }^{239} \mathrm{U}$ fission over a wide range of excitation energies. Signals from the detector were processed in two steps. At the first step, the masses and energies were reconstructed in an iterative procedure with corrections for the pulse height defect and for the emission of prompt neutrons from fission fragments. The resulting energy, mass, and angular distributions were found to be in good agreement with data presented in [8]. The mean total kinetic energy value was found to be $169.8 \mathrm{MeV}$ against $170.05 \mathrm{MeV}$ in [8]. But in such approach corrections for neutron emission performed based on the mass of nascent fragments only, without taking into account fission fragment excitation energy. This leads to a distortion in the region of heavy fragments spectrum, where neutron emission is energetically forbidden. Since this region is of special interest to present work, it is necessary to perform an additional processing of data using the energy calibration from the first step. Repeated iterations were done without the correction for neutron emission. This time the information about masses and energies appears to be incorrect for the majority of the fission fragments, but the high values region of the spectrum is correct.

\section{Results}

Two-dimensional spectrum with axis of fragment mass versus TKE for incident neutron energy of $5 \mathrm{MeV}$ is shown in Fig. 2 (left). Two-dimensional spectrum with axis of fragment mass versus TXE for the same neutron energy is shown in Fig. 2 (right).

The yields of nuclear-fission events where the heavy-fragment masses are 123 and $140 \mathrm{amu}$ versus the total kinetic energy (TKE) of fragments are shown in Fig. 3. Data for ${ }^{238} \mathrm{U}$ fission induced by 5 and $6.5 \mathrm{MeV}$ neutrons presented at the same plots for comparison. The distribution of total kinetic energies changes significantly with increasing of the excitation energy of the fissioning nucleus, especially in the high values region.

We would like to make special notes for several facts.

(i) The highest observed kinetic energy of fragment pairs where the heavy-fragment mass is $140 \mathrm{amu}$ have the same value for both values of the incident neutron energy $(6.5$ and $5 \mathrm{MeV})$, even though the production of fragments with greater value of TKE is energetically possible in the former case. This means that the entire energy introduced in the system goes to the excitation of fragments.

(ii) In the region of the highest values of $E k$ the yield of fragment pairs where the heavy-fragment mass is $140 \mathrm{amu}$ is one order of magnitude greater in the fission induced by $6.5 \mathrm{MeV}$ neutrons than for $5 \mathrm{MeV}$ neutrons.

(iii) The situation with fission fragments pairs where the heavy fragment mass is $123 \mathrm{amu}$ is different. For the incident neutron energy of $5 \mathrm{MeV}$ the measured maximum of TKE of such fragment pairs is $190 \mathrm{MeV}$, while for $E_{n}=6.5 \mathrm{MeV}$, it is by $10 \mathrm{MeV}$ higher, close to the value of the total implementable energy of $Q^{*}=202.7 \mathrm{MeV}$.

Figure 4 represents the difference between $Q^{*}$ and the maximum measured total kinetic energy of fragments as a function of the heavy-fragment mass for ${ }^{238} \mathrm{U}(\mathrm{n}, \mathrm{f})$ with $\mathrm{En}=5 \mathrm{MeV}$ and $\mathrm{En}=6.5 \mathrm{MeV}$. In the case of smaller incident neutron energy the maximum measured TKE reaches the value of $Q^{*}$ 


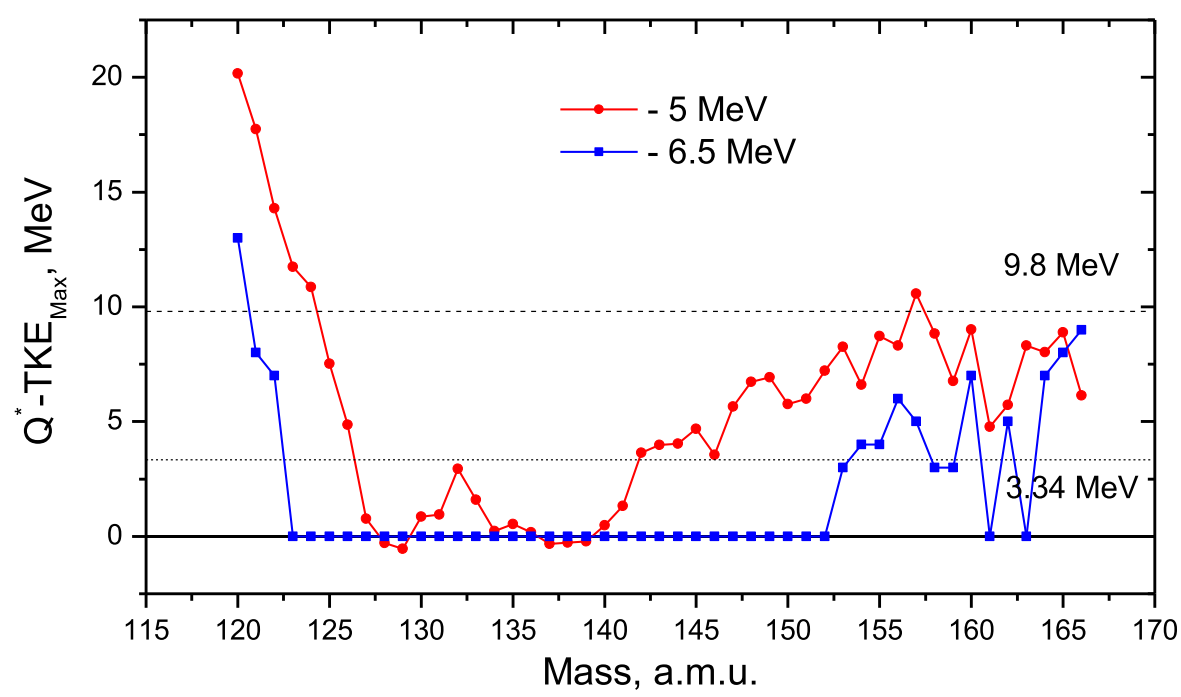

Figure 4. Mass dependence of available TXE value for $5 \mathrm{MeV}$ (red points) and $6.5 \mathrm{MeV}$ (blue points) incident neutron energy (data for ${ }^{238} \mathrm{U}(\mathrm{n}, \mathrm{f})$ ).

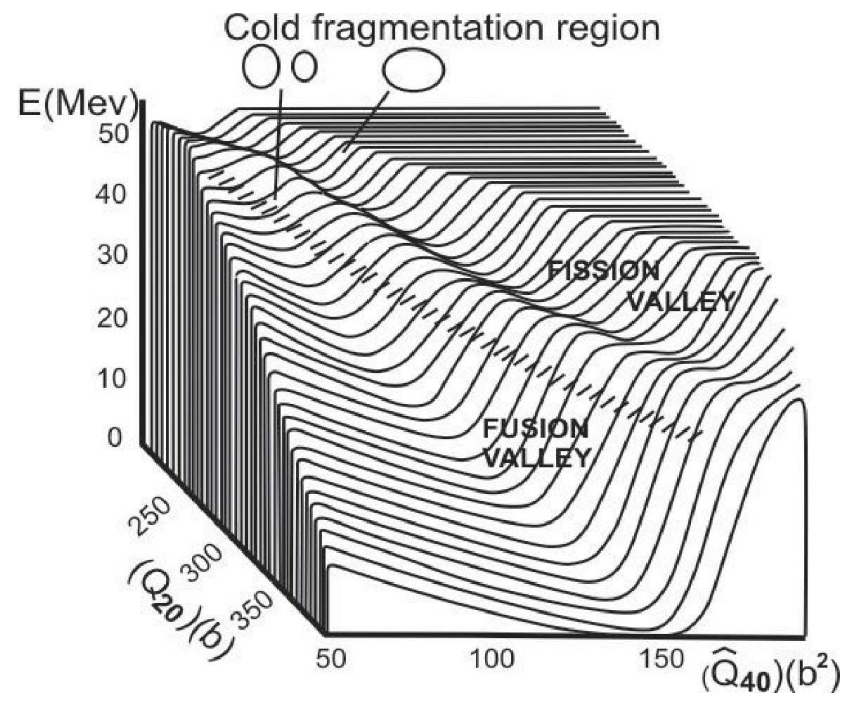

Figure 5. Potential-energy surface of ${ }^{240} \mathrm{Pu}$ versus the elongation of the nucleus, $\mathrm{Q}_{20}$, and the decrease in the thickness of its neck, $\mathrm{Q}_{40}$.

only for a small number of events where the heavy-fragment masses are 128, 129, and 134-139 amu. For $\mathrm{En}=6.5 \mathrm{MeV}$, this is true for all events where the heavy-fragment mass falls within the range of 123-152 amu. Despite of the fact that the increase in the excitation energy of the fissioning nucleus is only $1.5 \mathrm{MeV}$, the increase of maximum value of the measured total kinetic energy is $5-10 \mathrm{MeV}$. 
Figure 5 shows the surface of potential energy for the ${ }^{240} \mathrm{Pu}$ fissile nucleus [9]. Two valleys are clearly seen: the fission valley and the fusion valley. There is a potential barrier between them. Its height decreases with increasing of the fissioning nucleus deformation. At some deformation, the fissile system moves from the fission valley to the fusion valley, from one nucleus to two separate nuclei.

By this figure we are trying to understand qualitatively the results of our measurements in Figs. 3 and 4. The increase of $1.5 \mathrm{MeV}$ in the excitation energy of the fissile nucleus leads to a substantial increase in the yield of events in which $\mathrm{Ek}=\mathrm{Q}^{*}$. This is because the system then overcomes much more readily the potential barrier between the fission valley and the fusion valley, where the fragments already exist as individual nuclei. For those ways of nuclear mass separation for which, at the neutron energy of $5 \mathrm{MeV}$, the measured highest total kinetic energy of fragments already reached $\mathrm{Q}^{*}$, a further increase in the neutron energy only leads to an increase in the yield of such events. For a number of those ways of the separation of the nucleus where, at the neutron energy of $5 \mathrm{MeV}$, the highest measured values of Ek are smaller than $\mathrm{Q}^{*}$ by 5 to $10 \mathrm{MeV}$, the increase of $1.5 \mathrm{MeV}$ in the neutron energy leads to a substantial increase in the probability for overcoming the barrier in the case of events in which $\mathrm{Ek}=\mathrm{Q}^{*}$; at the total number of recorded fission events that is equal to about two millions, it also becomes possible to discover experimentally cold fragmentation for such ways of nuclear-mass separation.

\section{References}

[1] V.A. Khryachkov et al., Phys. At. Nucl. 57, 1154 (1994)

[2] A.A. Goverdovski et al., Phys. At. Nucl. 62, 901 (1999)

[3] A.A. Goverdovski et al., JETP Lett. 67, 793 (1998)

[4] C. Budtz-Jorgensen et al., Nucl. Instrum. Methods Phys. Res. A 258, 209 (1987)

[5] V.A. Khryachkov et al., Preprint No. 2740, Inst. Phys. Power Eng. (1998)

[6] V.A. Khriatchkov et al., Nucl. Instrum. Methods Phys. Res. A 394, 261 (1997)

[7] M.V. Dunaev et al., Proceedings of VIII Int. Seminar on Interaction of Neutrons with Nuclei, Dubna (2000), p. 363

[8] F. Vives et al., Proceedings of International Conference "Nuclear Fission and Fission-Product Spectroscopy", Seyssins, France (1998), p. 435

[9] J.F. Berger, M. Girod, and Gogny. Nucl. Phys. A 502, 85 (1989) 